\title{
On Term Translation based on Translational Examples
}

\author{
Gao Ran \\ Department of English, North China Electric Power University, Baoding, China. \\ 43784497@qq.com
}

Keywords: Terms, translation, ornithology

\begin{abstract}
A term is the carrier of ideological and cultural communication. Nowadays, terms are created at a speed in step with scientific and social development. Resulting from global integration, term translation plays an important role in international contexts. This paper takes a cue from translational examples of science and ornithology to uncover the essence and the universal law of term translation.
\end{abstract}

\section{Introduction}

The reason why China has accomplished a splendid civilization in history is that it took a constant embracing stance toward other cultures. Wang Guowei once said: "Parole represents thinking. Therefore, the import of new thinking to much degree means that of new words." The survival of Chinese civilization lies in its all-inclusive bearing and its humble attitude towards alien cultures even inferior ones. Ideological and cultural communication often co-occurs with translation practice. In China's history, three translation climaxes were present due to large-scale communication with other cultures. They are the spread of Buddhism from the late Han dynasty to Song, the import of Western subjects in late Ming and early Qing dynasties, and a swarm of Western learning in late Qing and early Republican era. Nowadays, the communication between China and the world is unprecedented in terms of scale, depth and frequency. Mountainous terms are created whenever communication occurs. In the same way the historical three translation climaxes in history left borrowed words in Chinese vocabulary, the contemporary communication results in a faster assimilation of foreign words. The significance of term translation is highlighted as foreign terms enriched Chinese vocabulary. This paper chooses term translation examples from science and ornithology with an aim to shed light on the universal law of term translation.

\section{Dissemination of Scientific Advances}

Translation activity concerned with scientific acquisition has a long history in some cultures. The history of science translation in China is a good source book with a considerable amount of information for further study. The Legacy of Islam gives many leads for the history of scientific translations from Arabic culture. For other cultures, the information in histories is less specially referred to, and there is much scope for enthusiastic researchers.

Scientific knowledge is more likely to cross borders of neighboring countries in the form of translation. Ancient China was once such a highly-developed kingdom. Advanced scientific and technical ideas written in books in ancient China were spreading in neighborhoods, from which Japan benefited a lot. The multi-storey pagoda came to Japan with Buddhism from China in the 6th century. A five-storey pagoda in Kyoto takes on architectural features learned from China. Through the Silk Road, the Four Great Inventions in ancient Chin and other techniques were introduced to Europe. After the Sino-Japanese War of 1894, the surprising rise of the once small and humble country like Japan shattered the arrogance and rigidness of Chinese people. As a result, Chinese government and scholars resorted to Japan for the latest science subjects and technical skills. Numerous terms rushed into 
Chinese academia during that period. The interaction between China and Japan is not an exception. The interplay of Britain and France is more complicated and thought-provoking.

Quite often a figure of considerable stature (in the academia, religious circles, or officialdom) will serve as a translator for the purpose of acquiring knowledge. Nicholas Culpepper (1616-64) published his critical English version of Pharmocopoeia from Latin in 1649. His translation project was to demystify the medical and scientific information that was being withheld from ordinary people who had no command of Latin. Some scholars published their retranslation of scientific works because they thought the original translation as appropriation by authorities or as misinterpretation by the public. Dolet's free translation of Plato was a deliberate challenge to the conservative Catholicism of the French authorities and he was burnt in 1546 due to his heretic behavior.

Translation activity concerning scientific exchange in China is connected to many factors. There were translators earning some social status getting involved consciously and unconsciously. The period from late Qing dynasty to early Republic of China saw a declining society. The connection of translation with knowledge and of knowledge with power provoked a larger number of patriotic officials and intellectuals to dedicate in translation activity for national salvation. This quest for advanced science was sponsored by clerical groups as missionaries made use of translation activity to make friends with the higher-class people and to interest Chinese people. Missioners such as Matteo Ricci, translating on one's own or cooperating with Chinese intellectuals, introduced the latest scientific and cultural findings to China, in which way they made big progress in preaching Gospel in China. Cooperation between Chinese intellectuals and western missioners characterized a feature of scientific translation. Another feature goes to the official investment on the promotion of acquiring knowledge. In the late of Qing dynasty, a number of translation institutions were founded by central and local governments with a view to bridging the increasingly broadened gap between China and western countries.

\section{From VR to *R: Is there A Law?}

Scientific and technological concepts are coming out at such a rapid speed that people could barely handle. VR is one of the examples which quickly attract people's attention as soon as they come into being. Within one year since the concept was created, VR has ignited a range of discussion. Similarly, augmented reality, AR in short, is another technology which was put forward in 1990. AR is a kind of technology which applies real-time data from cameras to video. This technology aims to set up interaction between virtual reality and real world. AR is a follow-up concept of VR. VR, namely virtual reality, was started in 1960s. This technology is designed to create a simulation system which integrates multi-source information. This simulation system enables three-dimensional dynamic visual to interact with actual behaviors. Users could build and experience virtual reality with this simulation system. In brief, virtual reality leads users to a virtual world which is full of virtual settings and images. Augmented reality is used to add virtual information to the real world so that settings and images are equally virtual and real. Augmented reality was born based on the development of virtual reality. The relationship between these two concepts may shed light on the translation of augmented reality. So far, there are three translation versions for this term.

FengZhiwei claims that, terminology should conform to the system principle. For one thing, if concepts are on the same hierarchical level, their terms should reflect the underlined logic. For another thing, once a term is decided as a fundamental term, other derivative terms or compound ones should make use of this fundamental term. According to the system principle, the translation of augmented reality should not be made from nothing but be put in a hierarchical system which could provide referential data for this translation. In terms of concept, augmented reality is intimately bound to reality. They are both terms from simulation technology and augmented reality is subsequent to virtual reality. They are on the same technological level. Consequently, the translation of virtual reality could be an 
example for that of augmented reality. In fact, virtual reality has only one the Chinese correspondence "xunixianshi" and this translation has already been widely accepted. If the data from "xunixianshi" are fully collected based on the system principle, a best translation for augmented reality could be easily found, that is “** xianshi”. "Kuozengshijing” is eliminated because it does not the hierarchy with virtual reality.

According to dictionary, augmented means "added to or made greater in amount or number or strength”. Both "kuozeng” and "zengqiang” are proper to correspond to "augmented”. Furthermore, we need to check them against the concept connotation. In terms of definition, augmented reality refers to the application of virtual information to the real world with a view to achieving ultra-real sensory experience. In short, augmented reality could make the real world greater. Therefore, both "zengqiangxianshi" and "kuozengxianshi" are considered to be loyal since they both reflect essential features of the concept.

The reason why "hun he xianshi" is also dumped is that it does not conform to accuracy and loyal principles. First of all, there is a semantic gap. Semantically, "hun he" in Chinese is not synonymous to "augmented" in English. In addition, "hun he" could not give a clue to the essential features of the concept. It fails to indicate the ultra-real experience. At last, if back-translated, "hun he xianshi” will become "mix reality". As a matter of fact, mix reality is not a synonym of augmented reality. Instead, it is another concept in simulation technology. Theoretically, term nomenclature requires monosemy, namely, a term is used to correspond to one concept in one field. To avoid misunderstanding, "hun he xianshi" is best to be a translation for "mix reality”. Resultantly, two translations are favored compared with other options: zengqiangxianshi and kuozengxianshi. In fact, zengqiangxian is prevalently used by the mainland academia which kuozengxianshi is used in Taiwan.

Science and technology still develops at an astonishingly fast speed. Simulation technology has gone ever farther to produce more new concepts before we completely swallow terms such as "xunixianshi" and "zengqiangxianshi”. New terms are more easily to be translated because regulation has been set up in this field. In addition to mix reality, there is cinematic coming out on the same hierarchical level with virtual reality. It is natural to work out "yingxiangxianshi" to translate "cinematic reality". Summarizing the current terms in simulation technology, a term formation model could be generated. "* Reality” is an English term formation model which could wholesale terms. In Chinese, “** xianshi” is a corresponding term formation model.

Abbreviation is a common way in scientific and technological terms due to time-saving, space-saving, and user-friendly reasons. A bundle of terms in virtual reality technology is undergoing a shift to abbreviation. Reports from leading news agency prefer to give full names of technological terms and use abbreviations in later paragraphs. Abbreviations such as AR, VR are not uncommon in academic papers. Even MR(Mix Reality) and CR(Cinematic Reality) are nothing new. Some prescient authors even conclude these terms as R terms and R products for those depending on this technology. What's more, as virtual reality technology is gaining popularity, ${ }^{*} \mathrm{R}$ is a promising term formation model over “** xianshi”. “* $\mathrm{R}$ ” is going to replace “** xianshi” as a more economic and productive term formation model.

\section{Ornithology: a Doorway to Term Translation}

Although ornithological terms are just a small part of the terminology iceberg, they shed light on the universal rules of nomenclature. The nomenclature rationale may vary from tribe to tribe due to social and cultural factors. For the same object, people may choose different features as its nomenclature basis. Obviously, a term may undergo variation when is transferred from one language to another. Another issue in this section is many translational correspondences for one term. Theoretically, a term is corresponded to one translation counterpart. In fact, many terms are given more than one translation counterparts. Some may be accepted as co-parts due to historical heritage, but some translation 
counterparts are elusive, ambiguous. Etymological identification is a better way to eliminate unqualified translational options.

Visual Image Features Give a Clue. A hypothesis from evolutionary theory says people of different tribes have a similar thinking pattern. They base a similar logical rule to create their languages and exchange thoughts. It is the similar logical rule that makes communication among differing languages possible. A supportive example for this hypothesis lies in people's nomenclature behavior. The way of nomenclature is mostly the same all over the world in which people make use of visual functions to identify denominate features. Fan Shouyi discusses nomenclature rules from a cognitive perspective and he finds that sense organs play a significant role in nomenclature. He classifies image features captured by sense organs into to categories. One is called visual image features which are obtained by visual observations. The other is para-image features which are observed by sense organs such as hearing, touching, tasting, smelling etc. Visual image features primarily focus on shape, size and color of the object and secondarily on its composition, material and function. Sometimes other features such distance, position, whole-part relation are also mentioned.

Three noun ornithological terms are to exemplify to what extent visual image feature may influence term translation. A comparison of visual image features between English and Chinese is present in the table below according to Fan Shouyi's research. In the table, “=” represents quits-relation, and a pair of terms between English and Chinese with quits-relation are called "quits-terms". " $<>$ ” means the pair of terms has a corresponding relation. That is to say, this pair of terms has differences from a certain sense organ. Pairs of terms falling into this kind of relation are called "corresponding terms". It is concluded that image features of noun terms may experience variation during translation: non-variation and variation.

Table 1.Image features between English terms and Chinese terms.

\begin{tabular}{|c|l|c|l|l|}
\hline \multicolumn{1}{|c|}{ English Terms } & $\begin{array}{l}\text { Image } \\
\text { Feature } \\
\text { (English) }\end{array}$ & vs & $\begin{array}{l}\text { Image } \\
\text { Feature } \\
\text { (Chinese) }\end{array}$ & $\begin{array}{l}\text { Chinese } \\
\text { Terms }\end{array}$ \\
\hline $\begin{array}{l}\text { spoon-billed } \\
\text { sandpiper }\end{array}$ & shape & $=$ & shape & shao sui yu \\
\hline $\begin{array}{l}\text { black-faced } \\
\text { spoonbill }\end{array}$ & color, part, & $<>$ & color, part, & heimian pi lu \\
\hline shorebird & position & $\begin{array}{c}=< \\
\text { bin }\end{array}$ & position & $\begin{array}{l}\text { bin } \\
\text { niao/shuinia } \\
\text { o }\end{array}$ \\
\hline
\end{tabular}

People resort to the same perspective to observe objects. Consequently they may come to same denominating features. In ornithology, both English and Chinese identify image features such as shape, color, part, position and so on in naming birds. There is slim possibility for two languages to denominate one object with the same image feature. If there any, it is called non-variation. For instance, spoon-billed sandpiper in English and shaojiaoyu in Chinese is a pair of quits-terms. They both choose mouth shape as their denominating feature. The same case goes to shorebird and bin niao. In most cases, due to diversified perspectives and social-cultural contexts, people may choose different image features and language resources to denominate objects. Shorebird and shuiniao, for instance, is a pair of corresponding terms. Shorebird emphasizes shore as the bird's habitat while shuiniao highlights water. This pair of terms reveals differing logic when denominating the bird. Black-faced spoonbill and heilian pi lu capture the same image features: black face and long bill. But English and Chinese nomenclature differs in using langue resources. For English people, spoon is a very ordinary utensil so they use spoon to modify the bird's long spill. Nevertheless, in the eye of Chinese people, it bears a strong resemblance to a musical instrument pipa. This pair of corresponding terms points to social-cultural variation.

Etymology Has The Final Say. In nomenclature, monosemy is a fundamental principle, that is, one 
concept should be designated by one term. But in fact, the case is non uncommon that one concept is given more than one names. Various reasons are responsible for this phenomenon. One reason is that one is on the par with the other so they co-exist for a long time. Regional difference may also result in more names for one concept.

A survey reveals that spoon-billed sandpiper has two equivalents: shaozuiyu and shaozuiyao. The two equivalents differ in the headword. Hence, which headword is appropriate? A scrutiny of their Chinese meaning may be self-evident. According to the Chinese Dictionary, yu refers to a type of ten-colored birds which have long spills and legs. They don't have webbed feet. They usually live along river or in fields on fish, worms and shellfish. Yao is also found in the dictionary explained as a type of ferocious birds which look like eagles but smaller in size, and live on small birds. Birdlife International gives the description of spoon-billed sandpiper as: (It has) small stint with spatulate bill. (It uses) adjacent estuary or mudflat habitats as breeding sites. During winter it prefers mixed sandy tidal mudflats with uneven surface and very shallow water, mainly in the outermost parts of river deltas and outer islands. By comparison, shaozuiyu bears a strong resemblance to spoon-billed sandpiper, thus shaozuiyao is dumped. The same way goes to the decision on sandpiper's translation. Anniao could be removed from the candidate list because it is seldom used. Literally, both shuiniao and binniao are considered as loyal translation. Based on semantic analysis, shuiniao focuses on the wetland where birds inhabit while binniao emphasizes that birds live in mud flat. Semantically, these two equivalents don't have essential differences and they are synonymous to each other. Finally, a conclusion may be achieved that sandpiper has two con-existent translations.

\section{Conclusions}

In this paper, practical examples from technology and ornithology are taken as a threshold to the universal law governing term translation. A scrutinize of those example plays a role in modern term translation practice. In order to promote term translation quality and validity, a consensus should be made. Firstly, a complete term formation pattern should be established to respond to the rapid-changing terms. Secondly, taking the fact that noun terms account for a large part in some fields, translators should probe for translation principles and criterion for noun terms. At last, situation varies from one term translation to another. Those terms which have more than one equivalent translation should be treated differently depending on their specialties. Inferior translated terms should be eliminated and superior ones are promoted. Meanwhile, co-existent translated terms may be kept when they are coming from regions such as Taiwan, Hong Kong and the Mainland.

\section{Acknowledgments}

This study was supported by the Fundamental Research Funds for the Central Universities (No.2015MS74).

\section{References}

[1] Fukang Chen. China’s Translation History. People’s Press, 2010.

[2] Yan Zhang. Essentials on Scientific Term Translation. Xinjiang University Press, 2008.

[3] Pinchuck, et al. Scientific and Technical Translation. Westview Press, 1977.

[4] Pearson, J. Terms in Context. John Benjamins, 1998.

[5] Xishan Zhou. Guowei Wang’s Works on Literature and Aesthetics. Beiyue Press, 1987.

[6] Zhiwei Feng. Introduction to Modern Terminology. Beijing: Shangwu Press, 2011. 